\title{
Erratum
}

\section{W. Helfrich: Possibility of Electrical Conduction in Filled Bands}

\author{
Z. Physik 205, 440 (1967)
}

\section{Received January 29, 1968}

It has been proposed in the discussion that the energy balance in an electric field is satisfied simply by momentum changes of the neutral particle causing the electron drag. Such an interpretation is not possible, as may be readily seen in the ring model. Here an electron keeps its wave vector when accelerated by a field, thus the total wave vector of the system consisting of the neutral particle and the electrons will also be conserved. Therefore, a change in the wave vector of the neutral particle requires that at least one electron alters its wave vector. Because of the exclusion principle this electron has to be excited into an empty band.

As to the calculations we found that the result for $\{\langle 0|N v| 2\rangle+\langle 2|N v| 0\rangle\}$ has to be corrected to give approximately

$$
\left[\frac{f_{0}}{2 \varepsilon_{f}(\pi / a)}\right]^{2} c \ln \frac{\hbar^{2} \pi \kappa_{f}}{\mu a|V|} .
$$

We wish to emphasize, however, that repeated checks and further simplifications of the model confirmed that $(N \bar{v})_{2}$ can be made nonzero. Only two possible explanations for this admittedly strange result appear to be left: (1) Divergence of the perturbation calculation in higher than second order. (2) Excitation of electrons into the conduction band by the combined action of the neutral particle and the electric field.

\author{
Dr. W. HeLFRICH \\ RCA Laboratories \\ Princeton, New Jersey 08540, USA
}

